\title{
Is the semi-regular variable RU Vulpeculae undergoing a helium-shell flash?^
}

\author{
S. Uttenthaler ${ }^{1}$, R. Greimel ${ }^{2}$, and M. Templeton ${ }^{3}$ \\ 1 University of Vienna, Department of Astrophysics, Türkenschanzstraße 17, A-1180 Vienna, Austria; \\ e-mail: stefan.uttenthaler@univie.ac.at \\ 2 IGAM, Institut für Physik, Universität Graz, Universitätsplatz 5/II, 8010 Graz, Austria; e-mail: rgreimel@ gmail.com \\ 3 American Association of Variable Star Observers, 49 Bay State Road, Cambridge, MA 02138, USA; \\ e-mail: matthewt@aavso.org
}

Received 03 Jul 2015, accepted 09 Nov 2015

Published online later

Key words stars: AGB and post-AGB - stars: late-type - stars: evolution - stars: oscillations - stars: individual (RU Vul)

The semi-regular variable star RU Vulpeculae (RU Vul) is being observed visually since 1935. Its pulsation period and amplitude are declining since $\sim$ 1954. A leading hypothesis to explain the period decrease in asymptotic giant branch (AGB) stars such as RU Vul is an ongoing flash of the He-burning shell, also called a thermal pulse (TP), inside the star. In this paper, we present a CCD photometric light curve of RU Vul, derive its fundamental parameters, and test if the TP hypothesis can describe the observed period decline. We use CCD photometry to determine the present-day pulsation period and amplitude in three photometric bands, and high-resolution optical spectroscopy to derive the fundamental parameters. The period evolution of RU Vul is compared to predictions by evolutionary models of the AGB phase. We find that RU Vul is a metal-poor star with a metallicity $[\mathrm{M} / \mathrm{H}]=-1.59 \pm 0.05$ and an effective surface temperature of $T_{\text {eff }}=3634 \pm 20 \mathrm{~K}$. The low metallicity of RU Vul and its kinematics indicate that it is an old, low-mass member of the thick disc or the halo population. The present day pulsation period determined from our photometry is $\sim 108 \mathrm{~d}$, the semi-amplitude in the V-band is $0.39 \pm 0.03 \mathrm{mag}$. The observed period decline is found to be well matched by an evolutionary AGB model with stellar parameters comparable to those of RU Vul. We conclude that the TP hypothesis is in good agreement with the observed period evolution of RU Vul.

(c) 2006 WILEY-VCH Verlag GmbH \& Co. KGaA, Weinheim

\section{Introduction}

The asymptotic giant branch (AGB) phase of evolution of stars with low initial masses $\left(1.0 \lesssim M / M_{\odot} \lesssim 8.0\right)$ is the last stage in which the energy output of the star is dominated by nuclear fusion. The main source of energy is the H-burning shell that is quasi-periodically interrupted and shut down by the He-burning shell, which is violently ignited during Heburning shell flashes. These events are also called thermal pulses (TPs) and have a strong impact on many observable parameters of the star such as its luminosity, radius, temperature, mass-loss rate, etc. For a discussion of the evolution of stars on the AGB, see e.g. Habing \& Olofsson (2003).

The outer envelope of stars on the AGB pulsates on timescales of tens to hundreds of days, thereby leading to brightness variations that are easily detectable. Thus, these stars belong to the semi-regular or Mira-like class of variables. Because the luminosity, radius, and temperature are altered during a TP, also the pulsation period is expected to change considerably when the star undergoes a TP. Wood

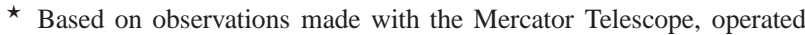
on the island of La Palma by the Flemish Community, at the Spanish Observatorio del Roque de los Muchachos of the Instituto de Astrofísica de Canarias
(1975) has first suggested that these period changes may result from the luminosity pulse produced by a TP. Wood \& Zarro (1981) substantiated this suggestion by comparing their AGB evolutionary models to the observed period evolution of a few Miras. Indeed, a number of Mira and semi-regular variable (SRV) stars are known to undergo significant period changes on timescales of decades to centuries (Zijlstra, Bedding \& Mattei 2002; Templeton, Mattei \& Willson 2005).

One SRV with known declining pulsation period is RU Vulpeculae (RU Vul; Zijlstra \& Bedding 2002). RU Vul's long-term light evolution from 1935, when visual observations began, to 2007 was analysed by Templeton, Willson \& Foster (2008). They find that the pulsation period started to decline around 1954, accompanied by a decrease in amplitude and an increase in average visual magnitude. The behaviour of RU Vul was compared by Templeton et al. (2008) to that of Mira-type variables that show similarly strong period changes. In fact, the early light curve of RU Vul shows a quite regular variability similar to Mira variables, only the amplitude was slightly too small to assign it to that class. Recently, Uttenthaler et al. (2011) obtained a high-resolution optical spectrum of RU Vul to search for lines of the element technetium (Tc), an indica- 
tor of the deep mixing event called a third dredge-up (TDU) that is expected to follow a few centuries after a TP (Vassiliadis \& Wood, 1993). No traces of Tc were found in the spectrum of RU Vul. However, this does not strictly exclude the possibility of a TP going on in that star.

In recent years, the pulsation amplitude of RU Vul has dropped to a level where it is hardly detectable by visual estimates. We therefore decided to perform CCD photometry to derive a reliable present-day pulsation period and amplitude in several photometric bands. In this paper, we present the results of these observations and determine some fundamental parameters of RU Vul. Most importantly, we test the prediction of the period evolution made by AGB evolutionary models with the help of the almost 80 years of visual light curve available for RU Vul.

The star has been assigned to the extended (thick) disc population of the Milky Way galaxy by Mennessier et al. (2001), based on its kinematics. We use the available information and our results to further check the population membership of RU Vul.

The paper is structured in the following way: In Sect. 2 we present the observations that were used for this study; in Sect. 3 the data are analysed and results are presented; finally, in Sect. 4 the results are discussed before conclusions are drawn in Sect.5.

\section{Observations and data reduction}

RU Vul is being monitored visually since 1935, and a complete visual light curve of the star is available from the AAVSO database (Henden, 2013). We downloaded all available data from 18 January 1935 to 15 July 2013. These data were averaged to ten-day bins, which were then analysed with the weighted wavelet Z-transform (wwz) algorithm developed by Foster (1996). We followed the procedure outlined in Templeton et al. (2005) to analyse the timefrequency behaviour of RU Vul. The strongest peak between 90 and 170 days of period was adopted as the actual period.

Because in recent years the variability of RU Vul became hardly detectable by visual estimates, we decided to augment the available AAVSO data by CCD photometric observations. The observations were carried out using the $30 \mathrm{~cm}$ Zeiss BMK-75 refractive telescope at Lustbühel observatory operated by the University of Graz, Austria. The data were collected using a SBIG ST-11000M CCD camera equipped with an $\mathrm{H} \alpha$ interference filter and JohnsonCousins V, R and I glass filters between 26 September 2011 (JD 2455831.2) and 12 June 2013 (JD 2456455.5). Standard data reduction was done using IRAF1 and aperture photometry extracted using SExtractor. The nearby K0 star HD 347112 was used as a comparison star to convert the relative brightness measurements to actual magnitudes.

${ }^{1}$ IRAF is distributed by the National Optical Astronomy Observatories, which are operated by the Association of Universities for Research in Astronomy, Inc., under cooperative agreement with the National Science Foundation.
The individual measurements will be made available on the AAVSO web page.

Furthermore, we used the ASAS V-band photometry retrieved from the ASAS project website. The ASAS observations were obtained in the V band between 26 April 2003 (JD 2452755.9) and 03 November 2009 (JD 2455138.5).

The high-resolution optical spectrum of RU Vul that we use here to derive the effective surface temperature and metallicity was obtained on 4 July 2009 with the fibre-fed spectrograph Hermes mounted to the $1.2 \mathrm{~m}$ Mercator telescope operated on the island of La Palma (Spain) by the Institute for Astronomy of the University of Leuven, Belgium. The spectrograph is described in Raskin et al. (2010). The spectrum was initially obtained by Uttenthaler et al. (2011), for details we refer to that work. The spectrum covers the whole optical range from $\sim 377$ to $\sim 900 \mathrm{~nm}$ at a resolving power of $R=\lambda / \Delta \lambda=85000$. The typical signal-to-noise ratio is $\sim 200$ in the range used here $(\sim 700 \mathrm{~nm})$. The spectrum was continuum-normalised in the analysed range by dividing it by a spline function that was fit through a number of continuum points.

To derive the present-day spectral type, we also observed RU Vul on 15 June 2012 (JD 2456094) with the $80 \mathrm{~cm}$ Cassegrain telescope of the Vienna University Observatory using a DSS-7 spectrograph manufactured by SBIG. The visual phase was midway from maximum to minimum at the time of observations. DSS-7 is a grating spectrograph offering five slits with different widths, the narrowest of which provides for a dispersion of $\sim 0.55 \mathrm{~nm} / \mathrm{pixel}$, corresponding to a spectral resolving power of $R \approx 500$. An ST-7 CCD camera, also made by SBIG, was connected to the spectrograph to acquire the spectra. With this camera, the wavelength coverage is approximately $450-850 \mathrm{~nm}$. The 2D raw spectra were dark-subtracted and flat-fielded with dome flats. To extract the 1D spectra, a Gaussian was fit to the profile in spatial direction. The wavelength calibration was done using the sky emission lines by adopting a second-order polynomial to map pixel positions to wavelength values.

\section{Analysis and results}

\subsection{Photometric analysis}

Our own photometric observations as well as the ASAS photometric data were analysed with the help of the SciPy package in Python. A least-squares fit was applied to match a sine curve to the data. The phase, period, semi-amplitude, and zero point offset were left free to vary. Fig. 1 shows the light curve observed at Lustbühel observatory in the $\mathrm{H} \alpha, \mathrm{V}$, $\mathrm{R}$, and I bands. Also shown in the figure is the best-fit sine curve to the V-band data with a period of $108.0 \pm 0.5 \mathrm{~d}$. The light curve somewhat deviates from the sine shape and it is not strictly periodic, as already found by Templeton et al. (2008, their Fig. 1), which underlines the semi-regular

\footnotetext{
2 http://wwW.astrouw.edu.pl/asas/
} 


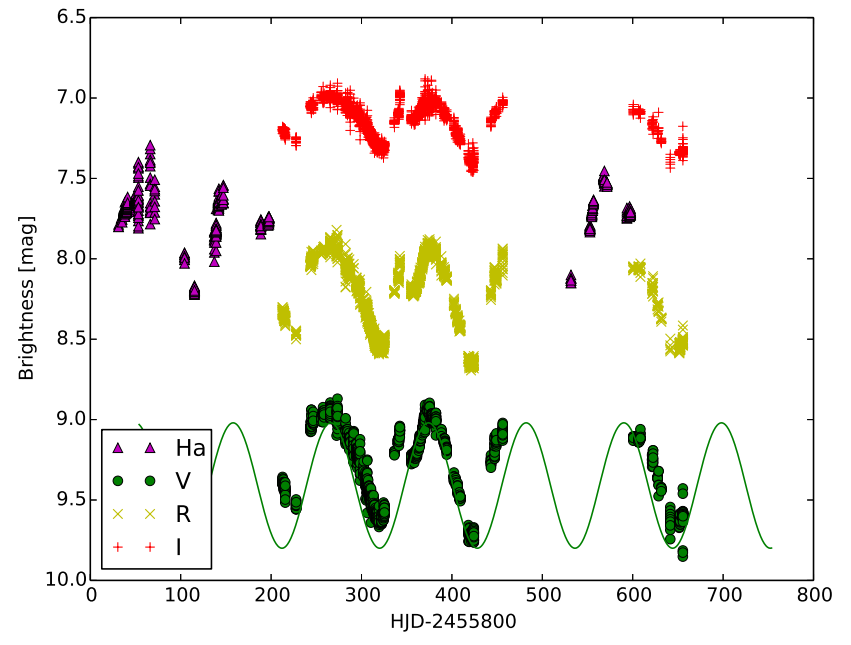

Fig. 1 Light curve of RU Vul obtained at Lustbühel observatory in the $\mathrm{H} \alpha$ (purple triangles), $\mathrm{V}$ (green dots), $\mathrm{R}$ (yellow crosses), and I (red plusses) bands. The best-fit sine curve to the V-band data with a period of $108.0 \mathrm{~d}$ is also shown.

behaviour of RU Vul. The phase shifts between the V, R, and I bands were found to be insignificantly different from zero.

The semi-amplitude of variability shows a clear dependency on the central wavelength of the used filter. Figure 2 shows the measured semi-amplitude as a function of central wavelength of the V, R, and I band filters. The filter transmission curves 3 are plotted as dotted lines in the same colours as the measured amplitudes, their values are to be read off on the right-hand y-axis. The $\mathrm{H} \alpha$ filter is not shown here because the amplitudes and their associated errors are derived directly from the observed data points, but the $\mathrm{H} \alpha$ light curve is not well sampled. The amplitude of variation in the I band is only about half as large as in the $\mathrm{V}$ and $\mathrm{R}$ bands.

Interestingly, the $\mathrm{V}$ band semi-amplitude found by our photometric observations indicates that the variability of RU Vul should be large enough to be easily detectable by visual eye estimates (Lebzelter \& Kiss, 2001), but it is hardly revealed. The range of reported brightnesses is in good agreement with that found by the photometric observations, but there is no regular variability in the light curve of the visual observations. Only if specific individual observers are picked out, a pulsation pattern is revealed. The reason for this is unclear to us.

Finally, also the ASAS V band light curve was analysed in the same way as our own photometric observations. A best-fit period of $107.6 \mathrm{~d}$ was found, a somewhat lower value than the $113.1 \mathrm{~d}$ quoted in the ASAS catalogue itself. However, inspection of the (folded) light curve shows that the value found by us appears to be a much better match to the observations, see Fig. 3. The pulsation period derived from ASAS is identical with that from our own obser-

\footnotetext{
3 http://ulisse.pd.astro.it/Astro/ADPS/
}

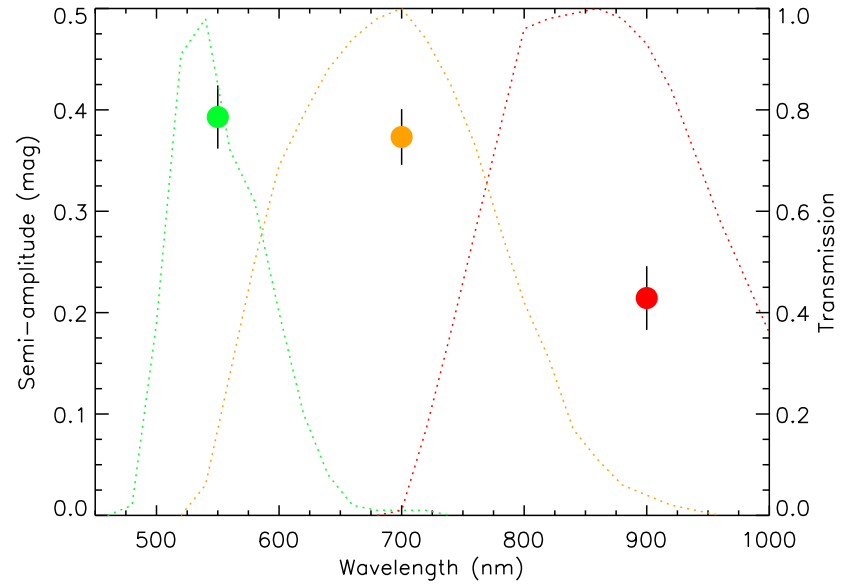

Fig. 2 Semi-amplitude of variation of RU Vul as a function of central wavelength of the V (green), R (orange), and I band (red) filters. The respective filter transmission curves are plotted as dotted lines in the same colours, the values are to be read off on the right-hand y-axis.

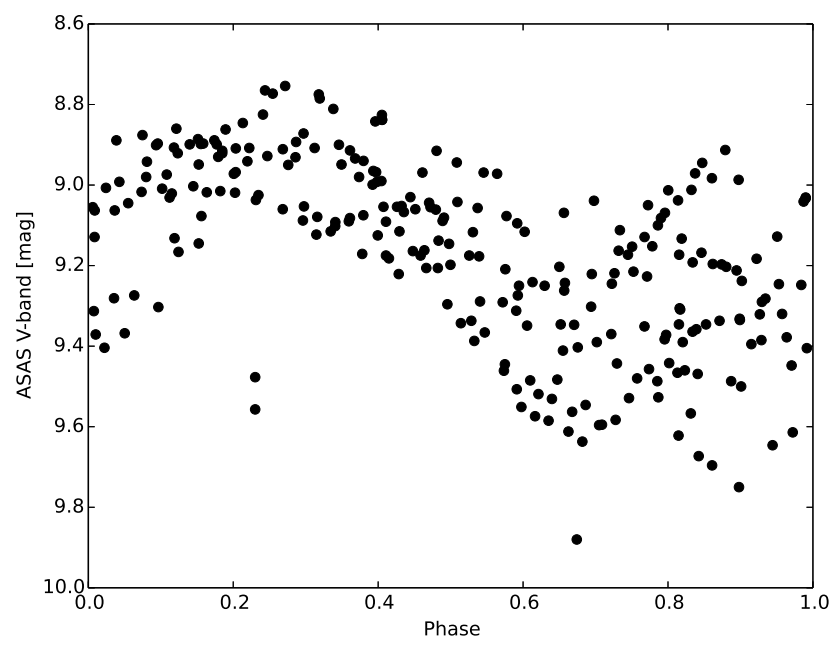

Fig. 3 Folded light curve of the ASAS photometric data, using a period of $107.6 \mathrm{~d}$.

vations within the uncertainties, indicating that the period has not significantly declined within the approximately six years between the ASAS and our observations.

\subsection{Spectroscopic analysis}

To derive the effective surface temperature and the metallicity of RU Vul from the high-resolution optical spectrum, we applied a similar approach as Valenti, Piskunov \& JohnsKrull (1998). These authors used an interpolation and fitting routine in a three-dimensional grid of synthetic spectra in $\left(T_{\text {eff }},[\mathrm{M} / \mathrm{H}], \log g\right)$ to derive the fundamental parameters of an M-type dwarf star. Here, we use an independent estimate of the surface gravity $\log g$ and do the interpolation and fitting only in a two-dimensional grid $\left(T_{\text {eff }},[\mathrm{M} / \mathrm{H}]\right)$.

To obtain an estimate of the surface gravity, we adopt the luminosity of $L=2830 L_{\odot} \pm 520 L_{\odot}$ given by Uttenthaler et 
al. (2011). This was derived from a period-magnitude relation for the K-band (sequence 1 for O-rich stars in Riebel et al. 2010, their Table 6) and a bolometric correction to the absolute $\mathrm{K}$ magnitude based on the $(J-K)_{0}$ colour (Kerschbaum, Lebzelter \& Mekul 2010, their Group A). The uncertainty on the luminosity was determined by combining in quadrature the uncertainties stemming from the 2MASS photometry (which is however minor) and the uncertainties quoted for the coefficients of the period-magnitude relation and the $\mathrm{BC}(\mathrm{K})$ correction. Since RU Vul probably is a member of the old, thick disc component of the Galaxy (Mennessier et al. 2001), we adopt a mass of $1 M_{\odot}$ for RU Vul. Considerations of the stellar life-time show that this is probably a good approximation. Furthermore, Uttenthaler et al. (2011) estimated a temperature of $3700 \mathrm{~K}$ from the mean spectral type (M3). Putting together the ingredients for stellar mass, luminosity, and temperature, we calculate $\log g=0.22$.

Given the surface gravity, we established a grid of model atmospheres with the COMARCS code (Aringer et al. 2009) and synthetic spectra based on this atmosphere grid. The grid covered the range $3500-4100 \mathrm{~K}$ in steps of $100 \mathrm{~K}$ and $[\mathrm{M} / \mathrm{H}]=-2.0$ to -0.5 in steps of 0.25 dex. Uttenthaler et al. (2011) had estimated the temperature at $3700 \mathrm{~K}$ and the metallicity to be close to $[\mathrm{M} / \mathrm{H}]=-1.5$, so the grid was constructed to bracket these approximate values. Only scaled solar abundances were used, assuming the set of solar abundances of Caffau et al. (2008). Excessive atomic (VALD; Kupka et al. 2000) and molecular (TiO, Schwenke 1998) line lists were used in the synthesis of the spectra with the COMA code (Aringer 2000). We used the range $698-715 \mathrm{~nm}$ for the fitting. This wavelength range is constrained by telluric lines that contaminate the observed spectrum at shorter and longer wavelengths. This piece of spectrum contains the $\mathrm{TiO} \gamma(0,0) R a, \gamma(0,0) R b$, and $\gamma(0,0) R c$ band heads, which are sensitive to the surface temperature of cool red giants. The metallicity is constrained simultaneously by the atomic lines that are present in that spectral range; in $\mathrm{RU}$ Vul, the $\mathrm{TiO}$ bands are not too strong to blanket all atomic lines, thus only one piece of spectrum is required to derive both quantities 4 .

The spectra were synthesised with an original resolution of $R=3 \times 10^{5}$, assuming a micro-turbulent velocity of $2.5 \mathrm{~km} \mathrm{~s}^{-1}$. The synthetic spectra were smoothed to the resolution of 85000 of the observed spectrum and different values of macro-turbulent velocities were added to improve the fit. It turned out that a macro-turbulent velocity of $11 \mathrm{~km} \mathrm{~s}^{-1}$ provided the best fit, but that the precise choice had virtually no influence on the obtained best-fit combination of temperature and metallicity; it only had the effect of improving the quality of the fit. Following Valenti et al. (1998), we did the interpolation in $\log (1-f)$, where $f$ is the continuum-normalised flux, to improve the convergence

4 Valenti et al. (1998) used two separate pieces of spectrum, one dominated by the temperature-sensitive TiO bands, another one dominated by metallicity-sensitive atomic lines.

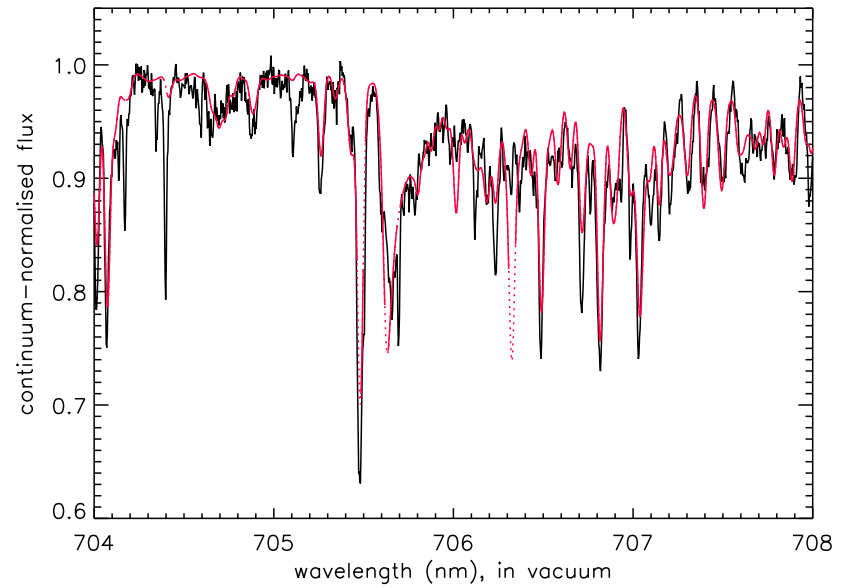

Fig. 4 Portion of the spectral range that was used to determine the metallicity and temperature of RU Vul. The observed spectrum is plotted as black line, the best-fitting interpolated synthetic spectrum as red line. Data points that were not included in the fitting procedure because they deviate by more than $10 \%$ from the observed flux are shown as dotted line. The broad feature starting at $705.6 \mathrm{~nm}$ is the TiO $\gamma(0,0) R a$ band head. Note the zoomed range on the $y$-axis.

of the fitting routine. A custom-made IDL routine was programmed, using the amoeba.pro simplex routine to find the minimum of a $\chi^{2}$ figure of merit. Because of inaccurate data in the atomic line list, in some lines the synthetic flux deviated considerably from the observed one, so we omitted wavelength points from the fitting procedure if $f_{\mathrm{obs}}-f_{\text {synth }}$ was larger than $0.1 f_{\mathrm{obs}}$, where $f_{\mathrm{obs}}$ is the observed flux and $f_{\text {synth }}$ the synthetic model flux. We found that the fitting routine converged reliably to the same best-fit combination of temperature and metallicity independent from the starting values within the grid.

Eventually, we found that the best-fit combination of temperature and metallicity or RU Vul is $T_{\text {eff }}=3634 \pm 20 \mathrm{~K}$ and $[\mathrm{M} / \mathrm{H}]=-1.59 \pm 0.05$. An illustration of the observed spectrum together with the best-fit interpolated synthetic spectrum is shown in Fig. (4) Overall, the fit is very satisfying and the determination of temperature and metallicity seem to be reliable.

With the newly determined temperature, the surface gravity changes to $\log g=0.18$ and one could do another iteration step to refine the metallicity and temperature by establishing a new model grid. However, since the change in $\log g$ is small and the features in the investigated spectral range are quite insensitive to $\log g$, we omitted another iteration.

Table 1 summarizes the most important stellar parameters derived here. 
Table 1 Main parameters of RU Vul.

\begin{tabular}{cc}
\hline Parameter & Value \\
\hline distance $(\mathrm{pc})$ & $2070 \pm 130$ \\
$\operatorname{luminosity~}\left(L_{\odot}\right)$ & $2830 \pm 520$ \\
$\log \left(g\left[\mathrm{cms}^{-2}\right]\right)$ & 0.18 \\
$T_{\text {eff }}(\mathrm{K})$ & $3634 \pm 20$ \\
{$[\mathrm{M} / \mathrm{H}]$} & $-1.59 \pm 0.05$ \\
radial velocity $\left(\mathrm{km} \mathrm{s}^{-1}\right)$ & -67.9 \\
pulsation period $(\mathrm{d})$ & $\sim 108.0$ \\
$<V>(\operatorname{mag})$ & 9.329 \\
$V$ amp. $(\mathrm{mag})$ & $0.393 \pm 0.031$ \\
$<R>(\mathrm{mag})$ & 8.287 \\
$R$ amp. $(\mathrm{mag})$ & $0.373 \pm 0.028$ \\
$<I>(\mathrm{mag})$ & 7.196 \\
$I$ amp. $(\mathrm{mag})$ & $0.214 \pm 0.031$ \\
\hline
\end{tabular}

\section{Discussion}

\subsection{Population membership}

Mennessier et al. (2001) assigned RU Vul to the extended (thick) disc. With a metallicity of $[\mathrm{M} / \mathrm{H}]=-1.59$, RU Vul is more metal-poor than most of the thick disc stars. However, it was shown by Ruchti et al. (2010) and Ruchti et al. (2011) that thick disc stars can have $[\mathrm{Fe} / \mathrm{H}]<-2$, although these are few objects. On the other hand, with its metallicity RU Vul is just at the peak metallicity of the metal-rich inner halo (Carollo et al., 2007). Note that we measured a general metallicity $[\mathrm{M} / \mathrm{H}]$, not $[\mathrm{Fe} / \mathrm{H}]$. Because metal-poor thick disc and halo stars have a general overabundance of $\sim 0.3 \mathrm{dex}$ in the $\alpha$-elements, to which also $\mathrm{O}$ and Ti belong, $[\mathrm{Fe} / \mathrm{H}]$ may be lower than $[\mathrm{M} / \mathrm{H}]$ in $\mathrm{RU}$ Vul. According to Reddy \& Lambert (2008), metal-weak thick disc stars cannot be assigned to a particular component.

Using the radial velocity of $-67.9 \mathrm{~km} \mathrm{~s}^{-1}$ and distance of $2070 \mathrm{pc}$ from Uttenthaler et al. (2011) and proper motion $\left(\mathrm{pm}_{\mathrm{RA}}, \mathrm{pm}_{\mathrm{DE}}\right)=(1.13,6.31) \mathrm{mas} / \mathrm{yr}$ from van Leeuwen (2007), we determine a space velocity of $(u, v, w)=$ $(-76.5,-33.6,39.8) \mathrm{km} \mathrm{s}^{-1}$. With this, RU Vul falls among the thick disc stars in the Toomre diagram of Bensby et al. (2005).

Its proximity to the Galactic plane $\left(b=-10^{\circ} 916\right.$, $z=-518 \mathrm{pc}$ ) speaks in favour of a disc membership, but it could also be a halo star that is close to the plane by chance. Thus, a clear population membership cannot be assigned to RU Vul, both a membership in the extended (thick) disc or in the metal-rich inner halo seem possible.

\subsection{Comparison to AGB evolutionary models}

Next we confront AGB evolutionary models with the observed period evolution of RU Vul. We selected a model from Vassiliadis \& Wood (1993) that well represents the main parameters of that star, namely the model with a mass of $1 M_{\odot}$ and a metallicity of $Z=Z_{\odot} / 16$, corresponding to $[\mathrm{M} / \mathrm{H}]=-1.20$, which is the model with the lowest metallicity in that set. The pulsation period in days was calculated

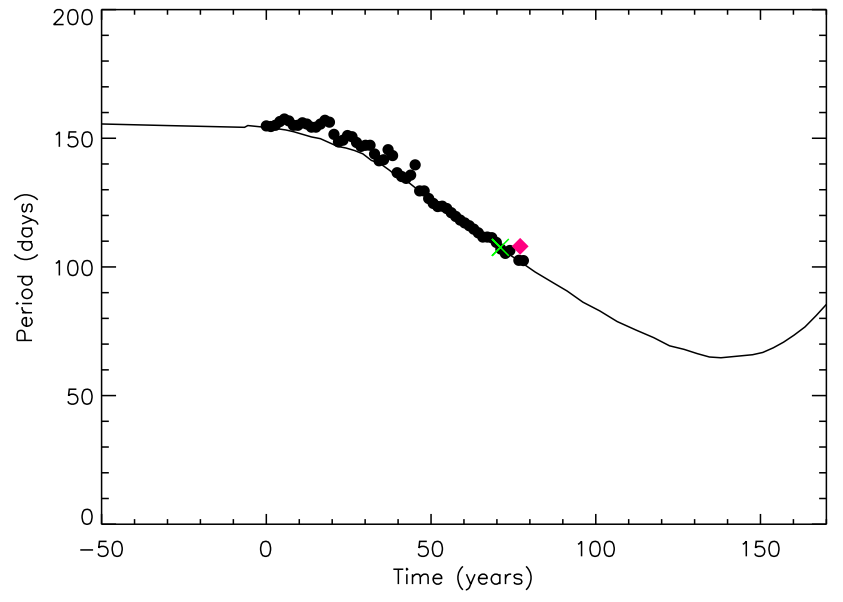

Fig. 5 Period evolution of RU Vul between 1935 and 2013 (black dots) and of the chosen AGB evolutionary model from Vassiliadis \& Wood $\left(1993 ; 1 M_{\odot}, Z=Z_{\odot} / 16\right.$, black line) at the beginning of the sixth TP of that model. Also shown is the period determination from the ASAS observations (green cross) as well as our own observations (red diamond).

from the total mass and the stellar model radius (derived from luminosity and surface temperature) using the equations given in Vassiliadis \& Wood (1993). From that model, we selected a thermal pulse cycle for comparison with the observations where the pulsation period before the onset of the TP (i.e. at the end of the quiescent H-burning phase) is close to the period of RU Vul before the period decrease, namely $\sim 155 \mathrm{~d}$. This is the case for the sixth TP (out of eleven TPs) of this model. We applied an arbitrary shift on the zero point of the time axis to match the observed period decline with that of the model. The result of this exercise is presented in Fig. 5] which also includes the period determination from the ASAS and our own data. The period evolution of RU Vul until 2007 has already been presented by Templeton et al. (2008, see their Fig. 2), here we only present an update of those data until 2013. The match between observed and predicted period evolution is remarkably good. We emphasize that except selecting this particular model and particular TP and the time zero point, we did not apply any fitting of the model to the data. However, it needs to be noted that a broad range of observed period behaviour may be fitted by different evolutionary models, thus a good match between observed and modelled period evolution is no solid proof that indeed a TP is going on in a given star.

Nevertheless, the model uncertainties on the short-time evolution are expected to be small because the models are quite robust regarding structural changes during a TP. Uncertainties in the treatment of mass loss and convection are not expected to alter the results significantly on a relatively short time scale of several decades.

Besides the pulsation period, which is a measure for the stellar radius, the TP scenario can be compared to other 


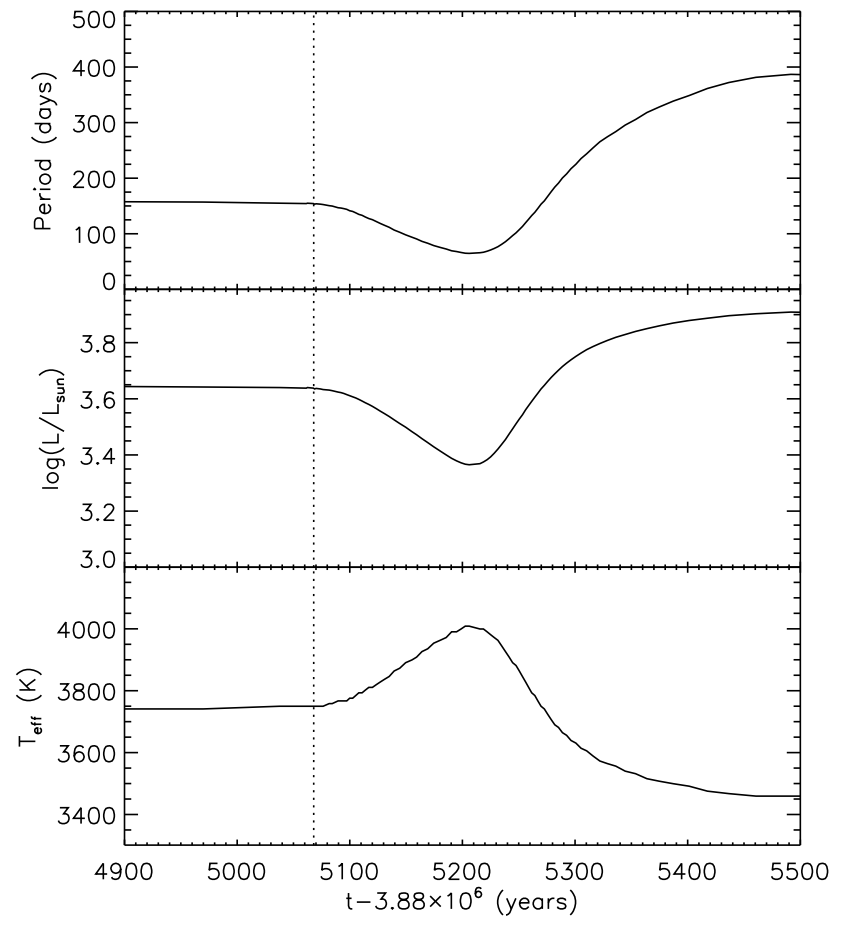

Fig. 6 Evolution of pulsation period (upper panel), luminosity (middle panel), and effective temperature (lower panel) of the model used in Fig. 5 during the first few hundred years after the onset of the 6th TP. The time is measured since the beginning of the AGB. The vertical dotted line marks the time zero point chosen for Fig. 5.

stellar quantities for further constraints. Figure 6 shows the evolution of the pulsation period, effective luminosity, and temperature of the adopted model for the first few hundred years after the onset of this particular TP. The model predicts that the pulsation period and the luminosity start to decrease when a TP begins, reaching minima of $\sim 65 \mathrm{~d}$ and $\sim 2300 L_{\odot}$ some 135 years after the onset of the TP, whereas the temperature starts to rise, reaching a maximum of $\sim 4000 \mathrm{~K}$. Later in the evolution of the TP, these trends reverse (Fig. 6). We inspect the available observational material of RU Vul to put further constraints on the TP hypothesis.

The luminosity of the model star at the end of quiet Hburning is $\sim 4350 L_{\odot}$, compared to $\sim 2830 \pm 520 L_{\odot}$ determined for RU Vul. This constitutes maybe the most serious difference between the actual properties of RU Vul and the adopted model, but note that the observed luminosity is based on a period-luminosity relation (using the period before the decline), not on an actually measured distance. Nevertheless, since the period decrease has commenced, RU Vul has brightened in the visual range because the cycle minima have become brighter, while maxima have roughly remained constant (Templeton et al. 2008, their Fig. 1). However, the visual (or V band) magnitude is not a good indicator for the bolometric luminosity of a red giant star because most of its light is emitted in the near IR. Unfortunately, no early IR observations of RU Vul are available.

The surface temperature of the model has risen from $\sim 3750 \mathrm{~K}$ to $\sim 3870 \mathrm{~K}$ by 78 years after the onset of the TP. This rise in temperature could lead to the observed increase in the visual brightness of RU Vul due to two effects. First, the emission maximum will be shifted to shorter wavelengths following the Wien law. Secondly, a higher temperature will decrease the molecular opacity in the visual range, which is mainly due to $\mathrm{TiO}$. The increase in temperature may also explain the observed decrease in pulsation amplitude (Templeton et al. 2008, their Fig. 2) because at a higher temperature the molecular $\mathrm{TiO}$ bands will be weaker.

An indication of an increase in temperature might come from spectral type classifications listed by Skiff (2013). RU Vul has been classified as M3e in 1897, M2 prior to 1943, and M4e in 1958, just as the period decline has started. We compared our own low-resolution observations (Sect.2) to the spectral type standards of Fluks et al. (1994) and find that the current spectral type is M0. This may indicate that the surface temperature of RU Vul has somewhat increased in recent decades, in agreement with expectations. We thus think that the brightness increase in the visual range is not in contradiction with the TP hypothesis.

As reported by Uttenthaler et al. (2011), no Tc has been detected in the atmosphere of RU Vul. Technetium is expected to be found on the surface of AGB stars that underwent a TDU, a deep mixing event that is thought to occur after powerful TPs. The simplest explanation for the absence of Tc is that no TDU event has (yet) taken place in RU Vul, or that the star has too low a mass to ever undergo TDU. On the other hand, very few seed nuclei are available at low metallicity so that the light s-process elements such as Tc are only very little enhanced, in favour of the heavy s-elements (Busso, Gallino \& Wasserburg 1999). However, also the singly-ionized lines of $\mathrm{Ba}$ at $649.87 \mathrm{~nm}$ and of $\mathrm{La}$ at $652.89 \mathrm{~nm}$ indicate that these s-elements are not enhanced in strength. Another effect of TDU would be the enrichment of the atmosphere with carbon, which would quickly lead to a $\mathrm{C} / \mathrm{O}$ ratio by number in excess of unity at low metallicity. Since RU Vul is clearly oxygen-rich, as signalled by the presence of $\mathrm{TiO}$ bands, we conclude that TDU has not occurred in RU Vul.

Finally, there are also other mechanisms that have been proposed to be able to lead to relatively quick changes of pulsation period in red giant stars. Zijlstra et al. (2004) propose that a possibly chaotic feed-back between molecular opacities, pulsation amplitude, and period can cause an unstable period in stars with a $\mathrm{C} / \mathrm{O}$ ratio very close to unity $(\mathrm{SC}$ and CS spectral types), as small changes in the temperature in the atmosphere can cause large changes in the molecular abundances, and hence the opacities. This mechanism can be excluded to be at play in RU Vul because the $\mathrm{C} / \mathrm{O}$ ratio is clearly below unity. Furthermore, Yaari \& Tuchman (1996) and Lebzelter \& Wood (2005) identify a feedback mech- 
anism between the pulsation and the stellar entropy structure, which can lead to mode switching. However, there are no detailed predictions as to how these mechanisms could be identified observationally.

\section{Conclusions}

We find that the SRV RU Vul is a metal-poor $([\mathrm{M} / \mathrm{H}]=$ $-1.59 \pm 0.05), \operatorname{cool}\left(T_{\text {eff }}=3634 \pm 20 \mathrm{~K}\right)$ red giant star whose chemical and kinematic properties are in agreement with either a thick-disc or a halo membership. The pulsation period decrease observed in RU Vul is well described by evolutionary models of the TP-AGB matched to the properties of the star. A decrease of the pulsation period for the next few decades is expected from these models. Also other observables such as pulsation amplitude and spectral sub-type, a proxy for the surface temperature, are in agreement with expectations from an ongoing TP. This suggests that RU Vul is a good candidate for a star that currently undergoes a TP, though our work cannot provide a solid proof for this suggestion. The evolution of this star is worthwhile to follow to learn more about these short, critical phases close to the endpoint of low-mass stellar evolution. Finally, we propose that RU Vul would also be of interest to study the mass-loss mechanism in metal-poor, pulsating red giants.

Acknowledgements. SU acknowledges support from the Austrian Science Fund (FWF) under project P 22911-N16. We thank Stefan Meingast for the assistance in the observations at the Vienna University Observatory. We acknowledge with thanks the variable star observations from the AAVSO International Database contributed by observers worldwide and used in this research.

\section{References}

Aringer, B.: 2000, PhD thesis, University of Vienna, Austria

Aringer, B., Girardi, L., Nowotny, W., Marigo, P., Lederer, M.T.: 2009, A\&A 503, 913

Bensby, T., Feltzing, S., Lundström, I., Ilyin, I.: 2005, A\&A 433, 185

Busso, M., Gallino, R., Wasserburg, G.J.: 1999, ARA\&A, 37, 239

Caffau, E., Ludwig, H.-G., Steffen, M., Ayres, T. R., Bonifacio, P., Cayrel, R., Freytag, B., Plez, B.: 2008, A\&A 488, 1031

Carollo, D., Beers, T. C., Lee, Y. S., Chiba, M., Norris, J. E., et al.: 2007, Nature 450, 1020

Fluks, M.A., Plez, B., The, P.S., et al.: 1994, A\&AS 105, 311

Foster, G.: 1996, AJ 112, 1709

Habing, H.J.; Olofsson, H.: 2003, Asymptotic giant branch stars, Astronomy and astrophysics library, New York, Berlin: Springer

Henden, A.A., 2013, Observations from the AAVSO International Database, http://www.aavso.org

Kerschbaum, F., Lebzelter, T., Mekul, L.: 2010, A\&A 524, A87

Kupka, F., Ryabchikova, T.A., Piskunov, N.E., Stempels, H.C., Weiss W.W.: 2000, Baltic Astronomy 9, 590

Lebzelter, T, Kiss, L.L.: 2001, A\&A 380, 388

Lebzelter, T., Wood, P.R.: 2005, A\&A 441, 1117
Mennessier, M.O., Mowlavi, N., Alvarez, R., Luri, X.: 2001, A\&A 374,968

Raskin, G., Van Winckel, H., Hensberge, H., Jorissen, A., Lehmann, H., Waelkens, C., Avila, G., de Cuyper, J.-P., et al.: 2010, A\&A 526, A69

Reddy, B.E., Lambert, D.L.: 2008, MNRAS 391, 95

Riebel, D., Meixner, M., Fraser, O., Srinivasan, S., Cook, K., Vijh, U.: 2010, ApJ 723, 1195

Ruchti, G.R., Fulbright, J.P., Wyse, R.F.G., Gilmore, G.F., Bienaymé, O., Binney, J., Bland-Howthorn, J., Campbell, R., et al.: 2010, ApJL 721, L92

Ruchti, G.R., Fulbright, J.P., Wyse, R.F.G., Gilmore, G.F., Bienaymé, O., Bland-Howthorn, J., Gibson, B.K., Grebel, E.K., et al.: 2011, ApJ 737, 9

Schwenke, D.: 1998, Faraday Discussions 109, 321

Skiff, B.A.: 2010, Catalogue of Stellar Spectral Classifications, VizieR On-line Data Catalog

Templeton, M.R., Mattei, J.A., Willson, L.A.: 2005, AJ 130, 776

Templeton, M.R., Willson, L.A., Foster, G.: 2008, JAAVSO 36, 1

Uttenthaler, S., Van Stiphout, K., Voet, K., Van Winckel, H., Van Eck, S., Jorissen, A., Kerschbaum, F., Raskin, G., et al.: 2011, A\&A 531, A88

Valenti, J.A., Piskunov, N., Johns-Krull, C.M.: 1998, ApJ 498, 851

van Leeuwen, F.: 2007, A\&A 474, 653

Vassiliadis, E, Wood, P.R.: 1993, ApJ 413, 641

Wood, P.R.: 1975, in IAU Colloquium 29, Multiple Periodic Variable Stars, ed. W.S. Fitch (Dordrecht: Reidel), p. 69

Wood, P.R., Zarro, D. M.: 1981, ApJ 247, 247

Ya'ari, A., Tuchman, Y.: 1996, ApJ 456, 350

Zijlstra, A.A., Bedding, T.R.: 2002, JAAVSO 31, 2

Zijlstra, A.A., Bedding, T.R., Mattei, J.A.: 2002, MNRAS 334, 498

Zijlstra, A.A., Bedding, T.R., Markwick, A.J., et al.: 2004, MNRAS 352,325 